\title{
Momentum conservation in Multi-Level Multi-Domain (MLMD) simulations
}

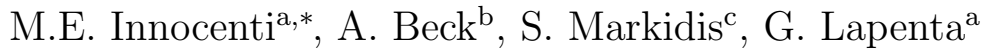 \\ ${ }^{a}$ Center for Plasma Astrophysics, Department of Mathematics, K.U.Leuven, \\ Celestijnenlaan 200B, B-3001 Leuven, Belgium \\ ${ }^{b}$ Laboratoire Leprince-Ringuet - Ecole polytechnique, CNRS-IN2P3, France \\ ${ }^{c}$ PDC Center for High Performance Computing, KTH Royal Institute of Technology, \\ Stockholm, Sweden
}

\begin{abstract}
Momentum conservation and self-forces reduction are challenges for all ParticleIn-Cell (PIC) codes using spatial discretization schemes which do not fulfill the requirement of translational invariance of the grid Green's function. We comment here on the topic applied to the recently developed Multi-Level Multi-Domain (MLMD) method. The MLMD is a semi-implicit method for PIC plasma simulations. The multi-scale nature of plasma processes is addressed by using grids with different spatial resolutions in different parts of the domain.
\end{abstract}

Keywords: Particle-In-Cell, Adaptive, Implicit, MLMD, self-forces, momentum conservation

\section{Introduction}

Momentum conservation and self-forces reduction are challenges for all Particle-In-Cell (PIC) codes [1] relying on non-uniform grids. Examples of such grids are the adaptive ones generated in PIC Adaptive Mesh Refinement (AMR) codes [2, 3], where the grid spacing is dynamically adjusted, and unstructured grids [4], where the connectivity is irregular.

\footnotetext{
${ }^{*}$ Corresponding author

Email addresses: mariaelena.innocenti@wis.kuleuven.be (M.E. Innocenti), beck@llr.in2p3.fr (A. Beck), markidis@pdc.kth.se (S. Markidis), giovanni.lapenta@wis.kuleuven.be (G. Lapenta) 
We recently introduced the Multi-Level Multi-Domain method, a semi-implicit [5] method for plasma PIC simulations which breaks the total domain into multiple grids resolved with different temporal and spatial resolutions $[6,7,8]$. Multiple resolution levels allow one to address the many scales emerging in plasma processes at a reduced computing cost. The Implicit Moment Method (IMM) is used at all grid levels. With the IMM, very different temporal and spatial resolutions can be used at the different grid refinement levels while satisfying the stability constraints of the method. The execution times may be tens of times lower than those of comparable uniform mesh simulations in 2D3V scenarios where high resolution jumps between the levels are used [9]. This note intends to clarify two basic points of the MLMD method. First, it addresses the issue of momentum conservation and self- forces generation, a fundamental challenge for adaptive methods. Second, it clarifies one of the implementation choices of the method, i.e. the use of the specific projection operator described in Eq. 32-33 of Innocenti et al. [6] and recapitulated in Eq. 12 here. These two basic points are deeply related, as shown in this note. In Section 2, the textbook derivation for the temporal variation of momentum $\Delta \mathbf{P}$ is recalled, albeit in a simplified electrostatic case. In Section 3, the derivation is extended to the coarse and refined levels of a MLMD system. Conclusions are then drawn.

\section{Momentum conservation}

In the continuum, the electrostatic problem is posed via the Poisson's equation

$$
\nabla^{2} \phi=-4 \pi \rho
$$

where $\phi$ is the electric potential and $\rho$ the charge, subject to the boundary conditions $(\mathrm{BC}) \phi_{\partial V}=\phi_{0}$.

It is possible to split the total potential $\phi$ of Eq. 1 into a contribution $\phi^{\text {in }}$ due to the inner charges in absence of external forcing and a boundary condition term $\phi^{B C}$. The Poisson's problem is then recast to

$$
\left\{\begin{array}{l}
\nabla^{2} \phi^{i n}=-4 \pi \rho \\
\phi_{\partial V}^{i n}=0
\end{array}\right.
$$

and

$$
\left\{\begin{array}{l}
\nabla^{2} \phi^{B C}=0 \\
\phi_{\partial V}^{B C}=\phi_{0} .
\end{array}\right.
$$


The electric field $\mathbf{E}$ is then provided by the gradient of the potential. Conveniently for the discussion of momentum conservation, this operation is written as

$$
\mathbf{E}(\mathbf{x})=-\nabla \phi=\mathbf{E}^{B C}(\mathbf{x})+\int \mathbf{G}\left(\mathbf{x}-\mathbf{x}^{\prime}\right) \rho\left(\mathbf{x}^{\prime}\right) d \mathbf{x}^{\prime}
$$

where $\mathbf{G}\left(\mathbf{x}-\mathbf{x}^{\prime}\right)=4 \pi \nabla \mathcal{G}\left(\mathbf{x}-\mathbf{x}^{\prime}\right)$ and $\mathcal{G}\left(\mathbf{x}-\mathbf{x}^{\prime}\right)$ is the Green's function for the electric potential.

The total electric field is expressed as the sum of the boundary condition term $\left(\mathbf{E}^{B C}(\mathbf{x})=-\nabla \phi^{B C}\right)$ and of the contribution from the internal charge. The discretization to a grid reads

$$
\mathbf{E}_{g}=\mathbf{E}_{g}^{B C}+\mathbf{G}_{g g^{\prime}} q_{g^{\prime}},
$$

with $q_{g^{\prime}}=\rho_{g^{\prime}} V_{g^{\prime}} . \rho_{g^{\prime}}$ is the density in the cell $g^{\prime}$ and $V_{g^{\prime}}$ is the cell volume. $\mathbf{G}_{g g^{\prime}}$ is the discretization of $\mathbf{G}\left(\mathbf{x}-\mathbf{x}^{\prime}\right)$ to the grid. A generic labeling of grid points as $g$ is used, regardless of the problem dimensionality. The standard convention that repeated indices are summed is assumed.

The temporal variation in momentum $\Delta \mathbf{P}$ follows by summing the velocity contribution of each particle and using the expression for the electric field given above:

$$
\Delta \mathbf{P}=\sum_{p} m_{p} \Delta \mathbf{u}_{p}=\Delta t \sum_{p} \mathbf{E}_{p} q_{p} .
$$

$\mathbf{u}_{p}$ is the particle velocity, $m_{p}$ the particle mass and $\Delta t$ the time step. The electric field acting on a particle is given by $\mathbf{E}_{p}=\mathbf{W}_{g p} \mathbf{E}_{g}$, where $\mathbf{W}_{g p}$ is the interpolation function (Eq. 7 of Lapenta et al. [10]) from grid to particle quantities $(g p)$. Substituting Eq. 5 into Eq. 6, one gets for $\Delta \mathbf{P}$ the expression

$$
\Delta \mathbf{P}=\Delta t \mathbf{E}_{g}^{B C} \mathbf{W}_{g p} q_{p}+\Delta t q_{p} \mathbf{W}_{g p} \mathbf{G}_{g g^{\prime}} q_{g^{\prime}} .
$$

Particle density is accumulated to grid points as

$$
q_{g}=\mathbf{W}_{p g} q_{p}
$$

Therefore, under the critical assumption that the same interpolation function is used from grid to particle quantities and vice versa $\left(\mathbf{W}_{g p}=\mathbf{W}_{p g}\right)$, Eq. 7 becomes

$$
\Delta \mathbf{P}=\Delta t \mathbf{E}_{g}^{B C} \mathbf{W}_{g p} q_{p}+\Delta t q_{g} \mathbf{G}_{g g^{\prime}} q_{g^{\prime}}
$$


If the boundary condition term can be neglected, the remaining term is responsible for the generation of self-forces, i.e. the spurious forces that particles impart on themselves as a result of the discretization scheme used [11]. Birdsall and Langdon [12] remark that they go to zero under two conditions: if 1) $\mathbf{W}_{g p}=\mathbf{W}_{p g}$, used earlier in the derivation, and if 2) the Green's function for the grid is translationally invariant, i.e. $\mathbf{G}_{g g^{\prime}}=-\mathbf{G}_{g^{\prime} g}$, so that $q_{g} \mathbf{G}_{g g^{\prime}} q_{g^{\prime}}=0$. Since these conditions are easily broken in non uniform grids, corrective actions are often required: Colella and Norgaard [13] introduce a new charge deposition scheme to reduce self-forces close to the grid refinement boundaries of an AMR code, where they are stronger. Bettencourt [14] subtracts off the self-forces caused by the particles at the various nodes of an unstructured grid.

In the next section, we will try to understand how much momentum nonconservation and self-forces generation affect the MLMD system and if corrective actions need to be undertaken.

\section{Momentum conservation in MLMD methods}

In the definition of momentum conservation in the MLMD system, it is considered that particles are simulated at all grid levels, the coarse and the refined one. According to Eq. 9, particles experience self-forces as a result of the interaction with the grid they live in. The analysis of momentum conservation is therefore carried separately on the refined and on the coarse grid. Eq. 9 is applied to the refined $\left(\Delta \mathbf{P}_{l 1}\right)$ and to the coarse grid $\left(\Delta \mathbf{P}_{l 0}\right)$. The extra terms appearing in $\Delta \mathbf{P}_{l 0}$ as a result of grid interaction are then discussed in depth. A simple MLMD system is examined: only two resolution levels, a coarse (l0) and a refined (l1) level, are considered, with a refinement factor $R F$ between the two defined as $R F=d \mathbf{x}_{l 0} / d \mathbf{x}_{l 1}$. The same time step is used on both levels, $d t_{l 0}=d t_{l 1}=\Delta t$.

Before proceeding, it is useful to recall a difference between the MLMD method in its current implementation and dynamically regridding methods, i.e. methods where the grid frequently changes adaptively in response to local conditions. In the latter cases, in presence of sharp gradients, a large percentage of the simulated domain may be bordering areas where the resolution changes. Consequently, a large area of the simulation may need correction

for self-forces effects. In the current implementation of the MLMD method, instead, regridding is not employed: the refined grid position and resolution 
are decided at the beginning of the simulation. Also, even if the refined grid area may be large, the boundaries between the different resolution areas cover a small fraction of the simulated domain. Fig. 1 depicts a sketch of a MLMD system. In the case of the refined grid, the boundaries between the coarse and the refined grid (in red) and the neighboring area (in orange) affect a very small number of cells with respect to the total number of refined grid cells simulated. The overlap area, i.e. the coarse grid area simulated with both resolutions (light blue in Fig. 1), extends for a fraction $1 / R F^{2}$ of the total domain. This is a very low number of coarse grid cells, especially if a high $R F$ is used. In the $2 \mathrm{D} 3 \mathrm{~V}$ magnetic reconnection simulations presented in Beck et al. [7], Innocenti et al. [9], $R F=12$ and $R F=14$. In these two cases, less than $1 \%$ of the coarse grid area belongs to the overlap area. The refined grid area superimposes to larger portions of the coarser grid when different problems are simulated, see for example Innocenti et al. [6].

Both the grids are, by themselves, structured and translationally invariant. On each grid, the same interpolation function is used to interpolate between grid and particle quantities and vice versa. This is true also on the refined grid: the particles at its boundaries are generated according to information interpolated from coarse grid particles (Eq. 37 to 39 in [6]), but with a shape function that matches the local grid size. Particle moments are interpolated to the grid using the usual interpolation function having as support the local cell size.

With these premises, Eq. 9 reduces to

$$
\Delta \mathbf{P}_{l 1}=\Delta t \mathbf{E}_{g}^{B C} \mathbf{W}_{g p, l 1} q_{p} .
$$

in the refined grid, where $\mathbf{W}_{g p, l 1}$ is the interpolation function having as support the refined grid cell size. Eq. 10 is equivalent to the level of spurious forcing imparted in a single grid simulation by the application of boundary conditions. Therefore, since the MLMD method does not affect momentum conservation in the refined grid with respect to single level simulations, no correction activities are put in place in the refined grid. In the coarse grid, momentum conservation cannot be dismissed as easily due to the field projection operation (blue arrow in Fig. 1) which is undertaken for grid interlocking purposes. After projection, the electric field $\mathbf{E}_{P, g}$ on the coarse grid is:

$$
\mathbf{E}_{P, g}=\mathbf{E}_{N, g}^{B C}+\mathbf{G}_{g g^{\prime}} q_{g^{\prime}}+\alpha \wp_{g}\left[-\mathbf{G}_{g g^{\prime}} q_{g^{\prime}}+\mathbb{P}^{g_{l 1} \rightarrow g_{l 0}}\left(\mathbf{E}_{N, g_{l 1}}\right)\right],
$$




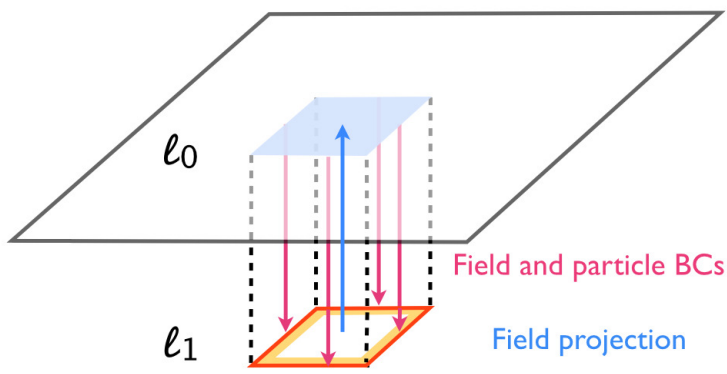

Figure 1: a sketch of a two level MLMD system. Grid interlocking operations (exchange of field and particle boundary conditions and field projection) are highlighted by red and blue arrows respectively. The areas potentially more severely affected by momentum non conservation are marked in colors: the refined grid boundaries and the neighboring area (red and orange) and the coarse grid overlap area (light blue).

Notice that, from now onwards (if not explicitly indicated), both the interpolation function and the discretized Green's function are assumed relative to the coarse grid, even if no labeling is used for the sake of readability. $\wp_{g}$ is a coarse grid operator identifying the overlap area and defined as:

$$
\wp_{g}=\frac{\sum_{g_{l+1}} \mathbf{W}_{g_{l}}\left(\mathbf{x}_{g_{l}}-\mathbf{x}_{g_{l+1}}\right)}{R F^{D}},
$$

where $D$ is the spatial dimensionality of the grid. The operator $\wp_{g}$ has value 1 in the coarse grid (CG) nodes corresponding to the inner part of the refined grid, $0<\wp_{g}<1$ in the CG nodes corresponding to the refined grid boundary cells and 0 in the CG area simulated only with low resolution.

The first term in the right hand side (RHS) of Eq. 11 is the now usual BC term (the notation $N$ stands for native, i.e. calculated on the grid in opposition to $P$, "after projection" [6]). The second RHS term includes the Green's function on the entire coarse grid, i.e. the coarse grid contribution in absence of feedback from the refined grid. The term in parenthesis accounts for how the electric field is calculated in the overlap area. There, the electric field solution is obtained by combining the "native" coarse grid solution (first term in parenthesis) and the refined grid solution "projected" to the coarse 
grid through the projection operator $\mathbb{P}^{g_{l 1} \rightarrow g_{l 0}}\left(\mathbf{E}_{N, g_{l 1}}\right)$ (Eq. (33) in [6]). We focus on two particular cases. If $\alpha=1$ ("projection by substitution"), the native electric field in the overlap area is completely substituted by the refined grid solution. If $\alpha=1 / 2$ ("projection by average"), the refined and native coarse grid fields are retained in equal parts.

Momentum variation on the coarse grid is then

$$
\begin{array}{r}
\Delta \mathbf{P}_{l 0}=\Delta t \mathbf{E}_{N, g}^{B C} \mathbf{W}_{g p} q_{p}+\Delta t q_{g} \mathbf{G}_{g g^{\prime}} q_{g^{\prime}}+ \\
\alpha \wp_{g} \Delta t\left[-q_{g} \mathbf{G}_{g g^{\prime}} q_{g^{\prime}}+\mathbb{P}^{g_{l 1} \rightarrow g_{l 0}}\left(\mathbf{E}_{N, g_{l 1}}\right) \mathbf{W}_{g p} q_{p}\right],
\end{array}
$$

The second RHS term is equal to zero, because the entire coarse grid is translationally invariant. The first term within parenthesis is however not, since the summation over the grid points $g$ is there restricted only to the nodes where $\wp_{g} \neq 0$. Notice, however, that the terms in parenthesis are opposite in sign and, presumably, close in absolute value, given the definition of $\mathbb{P}^{g_{l 1} \rightarrow g_{l 0}}\left(\mathbf{E}_{N, g_{l 1}}\right)$. A partial cancelation of the two is then expected. The partial and not total cancellation is due to small inconsistencies in charge distribution and in the electric fields across the levels, as discussed in Innocenti et al. [6], Sec. 6.3.

Also, the non conservation term may appear to be half in the case of projection by averaging with respect to projection by substitution. However, the terms in parenthesis, even if formally equal, are not necessarily so when $\alpha=1$ and $\alpha=1 / 2$. In fact, the field on the refined grid and the charge on the coarse grid do not necessarily evolve exactly in the same way with the two projection methods. It is thus necessary to compare the coarse grid moment evolution in the two cases to satisfactorily evaluate if momentum conservation really improves in the averaging case.

Fig. 2 shows the evolution in time of the coarse grid total momentum $\mathbf{P}_{l 0}$ normalized to the initial value $\mathbf{P}_{l 0,0}$ and calculated by summing all particle contributions. Three 1D3V cases are shown: a reference simulation (red line) of a Maxwellian plasma with no projection from the refined grid, a MLMD simulation with $\alpha=1 / 2$ (green line, AVE), and a MLMD simulation with $\alpha=1$ (blue line, SUB). In all the cases, the coarse grid BCs are periodic for fields and particles (the first term of Eq. 13 goes to zero). The other simulation parameters are identical and the same as the simulations shown in Innocenti et al. [6], Sec. 6.1: mass ratio $m_{i} / m_{e}=1836$, thermal velocity $v_{t h} / c=0.2$, with $c$ the speed of light, in all directions and for all species, coarse grid length $L_{x, l 0} / d_{e}=84$, with $d_{e}$ the electron skin depth, coarse 
grid resolution $d x_{l 0} / d_{e}=0.3$, time step $\omega_{p e} \Delta t=0.15$, with $\omega_{p e}$ the electron plasma frequency. A low $R F=4$ is used between the coarse and the refined grid. Notice that the choice of the $1 \mathrm{D}$ dimensionality and of a low $R F$ makes the test more challenging than in a $2 \mathrm{D}$, high $R F$ case, since the overlap area covers an higher percentage of the total coarse grid domain.

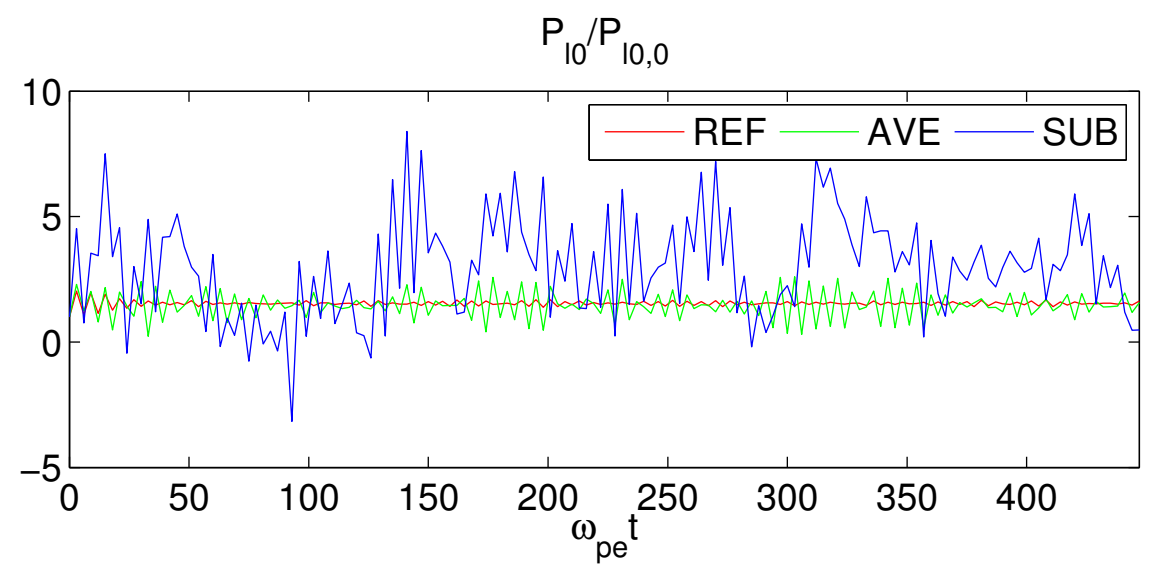

Figure 2: Time evolution of the total momentum $\mathbf{P}_{l 0}$ normalized to the initial value $\mathbf{P}_{l 0,0}$ for the coarse grid of Maxwellian plasma simulations with (red line) no coarse-refined grid interaction, (green line) projection by averaging and (blue line) projection by substitution. $\omega_{p e}$ is the electron plasma frequency.

In the case of projection by averaging (AVE) the momentum evolves in time rather similarly to the reference simulation (REF), with oscillation of controlled amplitude around the same average value. In the case of projection by substitution (SUB), instead, the oscillations are larger and the average value is higher.

Projection by average is thus shown to grant a significantly better momentum conservation than projection by substitution. As already for the refined grid, it is shown that the MLMD system (when projection by average is used) does not significantly degrade the level of momentum conservation in the coarse grid with respect to single grid simulations. Therefore, no correction activities are implemented in the coarse grid also.

\section{Conclusions}

We have analyzed the issue of momentum conservation and self-forces generation in the MLMD system, where the domain is simulated with dif- 
ferent grid levels using different spatial resolutions. Notwithstanding the presence of different resolution levels, all grids are translationally invariant and the same interpolation function is used for particle to grid quantities and vice versa. We show that, on the refined grid, non conservation of momentum only arises from boundary conditions, in a way similar to "standard" single grid simulations. Other terms are instead present on the coarse grid due to the field projection operations needed to ensure proper grid interlocking. There, however, satisfactory conservation of momentum is achieved, even in absence of specific correction activities, if projection is done by averaging of the refined and coarse grid electric field information rather than by completely neglecting the electric filed calculated locally on the coarse grid. Projection by averaging also has another advantage over projection by substitution: it allows the different grids a certain level of independent evolution. This means that the coarse and refined grid can more easily adjust to small scale processes accessible to the refined grid but not to the coarse. The simulation of processes at different scales (e.g.: ion scale processes on the coarse grid, electron scale processes on the refined grid) is the main achievement of the MLMD method, as shown in Beck et al. [7], Innocenti et al. [9]. Projection by average is fundamental in obtaining these results because it achieves two goals: the coarse and the refined grid solution are coupled and the coarse grid solution maintains a good level of momentum conservation. This result will be controversial in the larger context of AMR-type (local refinement) methods, which hold as a basic tenet that refined-grid solutions should be considered more accurate and thus should be used whenever possible. Such a philosophy presumes a strong preference for projection by substitution, in order that the coarse-mesh solution sees the full effect of the higher-accuracy fine-mesh solution. Our conclusions here are at odds with that philosophy because projection by averaging tends to act to limit the influence of the fine-mesh solution when considering momentum conservation.

\section{Acknowledgements}

This work has received funding from the European Unions Seventh Programme for Research, Technological Development and Demonstration under Grant Agreement No 284461 - Project eHeroes (www.eheroes.eu) and from the Interuniversity Attraction Poles Programme initiated by the Belgian Science Policy Office (IAP P7/08 CHARM). M.E.I. is funded by the FWO (Fonds Wetenschappelijk Onderzoek Vlaanderen) postdoctoral fellowship 
reference $12 \mathrm{O} 5215 \mathrm{~N}$.

[1] J. P. Verboncoeur, Plasma Physics and Controlled Fusion 47 (2005) A231.

[2] K. Fujimoto, R. D. Sydora, Computer Physics Communications 178 (2008) 915-923.

[3] J.-L. Vay, P. Colella, A. Friedman, D. Grote, P. McCorquodale, D. Serafini, Computer physics communications 164 (2004) 297-305.

[4] J. Hesthaven, T. Warburton, Journal of Computational Physics 181 (2002) $186-221$.

[5] H. Vu, J. Brackbill, Computer physics communications 69 (1992) 253276.

[6] M. Innocenti, G. Lapenta, S. Markidis, A. Beck, A. Vapirev, Journal of Computational Physics 238 (2013) 115 - 140.

[7] A. Beck, M. Innocenti, G. Lapenta, S. Markidis, Journal of Computational Physics 271 (2014) 430 - 443. Frontiers in Computational Physics Modeling the Earth System.

[8] T. Ponweiser, M. E. Innocenti, G. Lapenta, A. Beck, S. Markidis, Optimizing the multi level multi domain particle-in-cell code parsek2d-mlmd, in: http://www.prace-ri.eu/IMG/pdf/wp118.pdf.

[9] M. Innocenti, A. Beck, T. Ponweiser, S. Markidis, G. Lapenta, Computer Physics Communications 189 (2015) 47 - 59.

[10] G. Lapenta, J. Brackbill, P. Ricci, Physics of plasmas 13 (2006) 055904.

[11] J. Vay, P. Colella, P. McCorquodale, B. van Straalen, A. Friedman, D. P. Grote, Laser. Part. Beams 20 (2002) 569-575.

[12] C. K. Birdsall, A. B. Langdon, Plasma physics via computer simulation, Taylor \& Francis, 2004.

[13] P. Colella, P. C. Norgaard, J. Comput. Phys. 229 (2010) 947-957.

[14] T. Bettencourt, Plasma Science, IEEE Transactions on (2014). 